\title{
Network Formation with Endogenous Link Strength and Decreasing Returns to Investment
}

\author{
Chiu Ki So \\ Department of Economics and Finance, Hang Seng Management College, Shatin, Hong Kong; \\ jackieso@hsmc.edu.hk; Tel.: +852-3963-5089 \\ Academic Editor: Siegfried Berninghaus \\ Received: 21 September 2016; Accepted: 5 December 2016; Published: 8 December 2016
}

\begin{abstract}
We study the formation of networks where agents choose how much to invest in each relationship. The benefit that an agent can derive from a network depends on the strength of the direct links between agents. We assume that the strength of the direct link between any pair of agents is a concave function of their investments towards each other. In comparison with some existing models of network formation where the strength technology is a convex function of investment, we find that (i) the symmetric complete network can dominate the star architecture in terms of total utility; (ii) a dominating symmetric complete network needs not be stable; and, (iii) star and complete networks can be dominated by small-world networks.
\end{abstract}

Keywords: network formation; endogenous link strength; decreasing returns; small-world network

\section{Introduction}

The study of social networks is crucial for better understanding of issues ranging from job search [1] and diffusion of innovation [2] to the spread of contagious diseases [3] and civil protests [4]. The past two decades have witnessed a rapid growth in the economic literature on networks (for recent surveys, see Goyal [5] and Jackson [6]). An important branch of this literature examines how private incentives shape the formation of networks among a set of agents who behave strategically.

Starting with the pioneering work of Jackson and Wolinsky [7] and Bala and Goyal [8], most models of strategic network formation assume that the strength of the link between any two agents in a network is exogenously given. The efficient and stable network architectures vary with the marginal cost of forming a link relative to the benefit from a link. Take Jackson and Wolinsky [7]'s symmetric connections model and Bala and Goyal [8]'s two-way flow model with decay as examples, when the cost is relatively high, the empty network in which no one is connected to others is both efficient and stable. On the other hand, when the cost of forming a link is sufficiently low, the complete network (see the network on the right of Figure 1) in which all agents are directly connected turns out to be efficient and stable. The star (see the network on the left of Figure 1) is the stable and efficient network only when the marginal cost is at an intermediate level. As individuals do not take into consideration the externalities arising from their individual decisions, the centre of a star who bears most of the costs may not have an incentive to maintain a large number of links. Consequently, there exists a level of link cost such that the supported efficient network is not stable. Jackson and Wolinsky [7] term this phenomenon the tension between efficiency and stability (see Dutta and Mutuswami [9], Dutta and Jackson [10] and Jackson and van den Nouweland [11] for further discussion). 

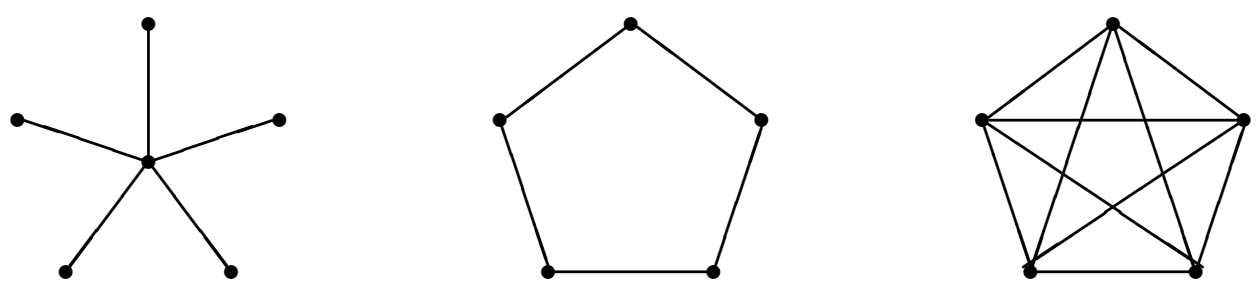

Figure 1. Star, wheel and complete network.

The symmetric connections model and the two-way flow model with decay neglect the importance of link strength which is a major focus of the network literature since Granovetter [1]'s seminal paper was published. To the best of our knowledge, Bloch and Dutta [12] and Deroïan [13] are the first to investigate models of strategic network formation with endogenous link strength: each agent allocates her (exogenously given) unit endowment to form links with other agents; the strength of the direct link between a pair of agents is assumed to be an additively separable and convex function of their investments towards each other. Efficient and stable architectures turn out to be a star in Bloch and Dutta [12] and a wheel (see the network in the middle of Figure 1) in Deroïan [13]. ${ }^{1}$ Results from the two models can be summarized as follows: (i) self-motivated agents will not diversify their investments and invest in no more than one link; (ii) efficient and stable networks are not clustered; and, (iii) there is no tension between efficiency and stability across architectures.

Convexity of the strength technology is the key assumption that leads to such outcomes. Intuitively, convexity implies increasing returns from investing in one and the same link and effectively rules out almost all complex networks. The assumption of convexity is quite reasonable in many contexts as discussed in Bloch and Dutta [12]. As they point out, increasing returns is suitable for the modelling of formal communication networks such as oil pipelines network and railways where fixed costs are significant. Yet, concavity of the strength technology might not be an inappropriate assumption, especially in the case of social networks, since even a relatively weak direct connection could be valuable [1]. Suppose an individual is searching for information on job vacancies from friends and relatives. A friend or relative is more likely to share her information with the individual if the strength of the links between them are strong. This provides incentive for individuals to maintain a small number of strong links. However, Granovetter [1] found that individuals receive crucial information from weak ties that they did not contact frequently, since weak ties enable them to reach populations that are not accessible via strong ties. In other words, valuable information does not necessarily come from the ties that one invests heavily in. This argument is also applicable to contexts such as searching for second hand goods and gathering information on sales via Facebook and Twitter. In such cases, individuals may follow a single Facebook page which consolidates information from other individuals. Then a star is formed. Alternatively, they may choose to exchange information directly if weak ties are sufficient for them to derive valuable information from others. Then a complete network will be formed. Therefore, the degree of concavity of the strength technology will determine which structure is going to emerge.

A complete characterization of efficient and stable networks for concave strength technologies seems analytically formidable. In the following, we first compare our paper with some recent network formation literature. Then we will show that, under concave strength technologies, a complete network

1 Differences arise because the two papers assume different flows of benefits. In Bloch and Dutta [12], the strength of link is the sum of the resources invested by the pair of agents. Stars arise because they can maintain a short distance between agents while each agent invests only in a single link. On the other hand, Deroïan [13] assumes the strength of link with respect to an agent is the resources invested by the same agent over that link. A wheel arises because it is the only architecture which allows each agent to be able to derive benefits from all other agents given that each agent invests only in a single link. 
can be stable and dominate all feasible star networks. Moreover, a dominating complete network may be unstable, thereby restoring the tension between efficiency and stability. Finally, with the help of a simple example, we show that a small-world network can dominate the star and complete networks.

The current paper can be considered as a variation of Bala and Goyal [8]'s two-way flow model with decay by assuming that the strength of links is endogenously chosen by the agents. Bloch and Dutta [12], Deroïan [13] and Feri and Meléndez-Jiménez [14] also extend Bala and Goyal [8] by endogenizing the strength of links. First, Bloch and Dutta [12] and Deroïan [13] assume for increasing returns to investment. The former is based on the two-way flow model while the latter is based on the one-way flow model. On the other hand, Feri and Meléndez-Jiménez [14] consider a dynamic model of network formation where the strength of links is the outcome of a coordination game instead of a function of the agents' direct investments. In Bala and Goyal [8]'s non-cooperative game, since the formation of links does not require the mutual consent between the pair of agents, it is natural to assume that the strength technology is additively separable $[12,13]$. However, when mutual consent is required as suggested by Jackson and Wolinsky [7], a complementary function seems to be more appropriate. Following this line of research, based on Jackson and Wolinsky [7]'s co-author model, Harmsen-an Hout et al. [15]'s model with endogenous link strength shows that small-world networks can also be supported as an equilibrium. Moreover, an efficient network need not be stable. The emergence of complex networks is uncommon in standard models of network formation unless heterogeneity across agents is introduced (e.g., Jackson and Rogers [16]). In contrast with Bloch and Dutta [12] and Deroïan [13], our paper completes the picture by showing that similar results can also be achieved when mutual consent is not required if decreasing returns to investment is assumed. ${ }^{2}$

\section{The Model}

We study a model which is similar to Bloch and Dutta [12] except for the specification of the strength technology. Let $\mathbf{N}=\{1,2, \ldots, n\}$ denote the finite set of agents where $n \geq 3$. We assume each agent is endowed with one unit of resource. Let $x_{i}^{j} \in[0,1]$ denote the investment by agent $i$ in the direct link with agent $j$. A strategy for agent $i$ is a vector $\mathbf{x}_{i}=\left(x_{i}^{1}, \ldots, x_{i}^{i-1}, 0, x_{i}^{i+1}, \ldots, x_{i}^{n}\right)$, where $\sum_{j \in \mathbf{N}} x_{i}^{j} \leq 1$. Let $\mathbf{x}=\left(\mathbf{x}_{1}, \mathbf{x}_{2}, \ldots, \mathbf{x}_{n}\right)$ be a strategy profile. The strength of the direct link $i j$ between any two distinct agents $i, j \in \mathbf{N}$ is a function of their investments towards each other and will be denoted as $\sigma_{i j}\left(x_{i}^{j}, x_{j}^{i}\right)$.

Given a strategy profile $\mathbf{x}$, the network which results can be denoted as an $n \times n$ matrix $g(\mathbf{x})$, where $g_{i j}=1$ if $\sigma_{i j}\left(x_{i}^{j}, x_{j}^{i}\right)>0$ and $g_{i j}=0$ otherwise. A path between agents $i$ and $j$ in a network $g$, denoted by $p(i j ; g)$, is a sequence of distinct nodes $i=i_{0}, i_{1}, \ldots, i_{K-1}, i_{K}=j$ such that $g_{i_{k} i_{k+1}}=1$ for all $k \in\{0, \ldots, K-1\}$. Let $\mathbf{P}(i j ; g)$ be the set of all paths connecting agents $i$ and $j$ in $g$. Two distinct agents $i$ and $j$ are connected in $g$ if and only if $\mathbf{P}(i j ; g) \neq\{\varnothing\}$. They are directly connected if the shortest path connecting them is a direct link. The three main assumptions of the model are as follows.

[Assumption 1] Strength Technology The strength of the direct link between any two distinct agents $i, j \in \mathbf{N}$ is

$$
\sigma_{i j}\left(x_{i}^{j}, x_{j}^{i}\right)=\phi\left(x_{i}^{j}\right)+\phi\left(x_{j}^{i}\right)
$$

2 The assumption of additively separability is not critical for the result of efficiency in our model. However, it is critical for the result of stability. For instance, a star is always Nash stable if mutual consent is required because a peripheral cannot benefit by deviating without another peripheral deviating simultaneously. Accommodating the idea of mutual consent requires an equilibrium concept which allows for a certain degree of cooperative behavior (e.g., pairwise stability). Readers who are interested in this line of research can consult Baumann [17] and Salonen [18]. Both papers are variations of Jackson and Wolinsky [7]'s symmetric connections model in their relaxation of the assumption of exogenous link strength. 
where (i) $\phi(0)=0$, (ii) $\phi(1)<\frac{1}{2}$, and (iii) $\phi(\cdot)$ is increasing and strictly concave. These assumptions imply that $\sigma_{i j} \in[0,1)$ for all $i, j \in \mathbf{N}$.

[Assumption 2] Reliability of a Path The reliability of a path between any two distinct agents $i, j \in \mathbf{N}$ in a network $g$ is given by the product of the strengths of the links along that path. Formally,

$$
r(p(i j ; g))=\sigma_{i i_{1}} \cdots \sigma_{i_{k-1} i_{k}} \cdots \sigma_{i_{K-1} j} .
$$

One could alternatively say that the strength of each direct link along a path defines the degree of decay of the path.

[Assumption 3] Utility We assume every pair of agents will only communicate through their most reliable path. Thus, the utility derived by an agent $i$ under network $g$ is the sum of the reliabilities of the most reliable paths connecting him to other agents and is formally denoted by

$$
u_{i}(g)=\sum_{j \neq i} \max _{p(i j ; g) \in \mathbf{P}(i j ; g)} r(p(i j ; g)) .
$$

Throughout this paper, the term connection shall refer to the most reliable path connecting a pair of agents.

Network $g$ dominates network $h$ if and only if the sum of utilities of all agents under $g$ is strictly higher than that under $h$. A network is efficient if it maximizes the sum of utilities derived by all agents and thus (weakly) dominates every other network. A network $g(\mathbf{x})$ is Nash stable if no agent has a strict incentive to unilaterally deviate from his strategy.

\section{Results}

The following two concepts will be used throughout the rest of the paper. A network is essential if and only if every link in the network lies along the most reliable path connecting some pair of agents. Essentiality implies that no link is redundant. A network $g(\mathbf{x})$ is budget exhausting if and only if $\sum_{j \in \mathbf{N}} x_{i}^{j}=1$ for all $i \in \mathbf{N}$. A budget exhausting network implies no waste of resources. The following Lemma shows that an efficient or Nash stable network must be essential and budget exhausting. It allows us to focus only on essential and budget exhausting networks in the following analysis.

Lemma 1. An efficient or Nash stable network is essential and budget exhausting.

We now define two special network structures. A complete network is symmetric if and only if every agent invests an equal amount towards all the other agents. Similarly, a star is symmetric if and only if the hub invests an equal amount towards each peripheral agent. We denote a symmetric complete network by $g^{s c}$ and a symmetric star by $g^{s s}$.

Proposition 1. If $A 1-A 3$ hold, then for all $\alpha \in(0,0.5)$, there exists $\beta \in(0, \alpha)$ such that, for all strength technologies such that $\phi(1)=\alpha$ and $\phi\left(\frac{1}{n-1}\right)>\beta$, the symmetric complete network dominates all star networks.

The proof exploits the idea that, under a concave strength technology, if an agent is directly connected with every other agent and communicates only through direct links, then equally splitting the endowment in all the direct links is optimal. This allows us to prove that the symmetric complete architecture dominates every other complete architecture and symmetric star architecture dominates every other star architecture. Finally, a direct comparison of the total utilities generated by the two networks delivers the result. Note that the sum of utilities of all agents in the symmetric complete network is

$$
U\left(g^{s c}\right)=n(n-1)\left[2 \phi\left(\frac{1}{n-1}\right)\right]
$$


Similarly, the sum of utilities of all agents in the symmetric star is

$$
U\left(g^{s S}\right)=2(n-1)\left[\phi(1)+\phi\left(\frac{1}{n-1}\right)\right]+(n-2)(n-1)\left[\phi(1)+\phi\left(\frac{1}{n-1}\right)\right]^{2}
$$

The comparison of these two networks relies crucially on two particular values of the strength technology $-\phi(1)$ and $\phi\left(\frac{1}{n-1}\right)$. The former is the maximum strength of a link an agent can ensure by unilateral investments. The latter is the strength from unilateral equal allocation of the unit endowment. Proposition 1 shows that, for a given value of maximum strength, as $\phi\left(\frac{1}{n-1}\right)$ increases, the symmetric complete network will tend to dominate the symmetric star. Rearranging $U\left(g^{s c}\right)>U\left(g^{s S}\right)$ gives

$$
2(n-1)^{2}\left[\phi\left(\frac{1}{n-1}\right)-\left(\frac{1}{n-1}\right) \phi(1)\right]>(n-1)(n-2)\left[\phi(1)+\phi\left(\frac{1}{n-1}\right)\right]^{2}
$$

The left hand side of (6) accounts for the difference in utilities generated by direct connections within the two network architectures. The right hand side of (6) accounts for the difference in utilities generated by indirect connections within the two network architectures. In particular, the term $\phi\left(\frac{1}{n-1}\right)-\frac{1}{n-1} \phi(1)$ effectively captures the gains from diversification due to equal split of endowments. If the strength technology is convex, this term will be negative and $g^{s c}$ cannot dominate $g^{s s}$. Hence, the higher the value of $\phi\left(\frac{1}{n-1}\right)-\frac{1}{n-1} \phi(1)$, the more likely it will be that the symmetric complete network architecture dominates the symmetric star architecture.

Proposition 2. If $A 1-A 3$ hold, then

(a) the symmetric complete network is Nash stable if $\phi(1)+\phi\left(\frac{1}{n-1}\right)<1 / 2$;

(b) the symmetric star network is Nash stable if $\phi(1)+\phi\left(\frac{1}{n-1}\right)>1 / \sqrt{2}$.

Proposition 2 provides a sufficient condition for the stability of the symmetric complete network and symmetric star. Concretely, any deviation from a symmetric complete network must involve replacement of some direct connections by an indirect connection. The resulting network cannot be essential if the highest possible reliability of the newly established indirect connection $-2 \phi\left(\frac{1}{n-1}\right)\left[\phi(1)+\phi\left(\frac{1}{n-1}\right)\right]$ - is less than the left over strength of the direct connection after deviating $-\phi\left(\frac{1}{n-1}\right)$. On the other hand, because all the peripherals in a star are investing all their endowments in the links with the hub, any deviation must be a replacement of indirect connections with direct connections. The resulting network will not be profitable if the reliability of the newly established direct connection, which cannot exceed half, is less than the reliability of the indirect connections in a symmetric star $-\left[\phi(1)+\phi\left(\frac{1}{n-1}\right)\right]^{2}$.

We make three observations: First, Proposition 1 and part (a) of Proposition 2 together show that, for a given value of $\phi(1)$, an increase in $\phi\left(\frac{1}{n-1}\right)$ tends to make the complete network dominant but unstable. The basic idea behind this feature is that a higher $\phi\left(\frac{1}{n-1}\right)$ has two different impacts. On the one hand, it has a positive impact on the gains from diversification which favors the dominance of $g^{s c}$ over $g^{s s}$. On the other hand, it also lowers the degree of decay of the indirect path which makes deviations from the symmetric complete network more likely to be profitable. A dominating symmetric complete network is unstable when the latter effect outweighs the former effect.

Second, according to part (b) of Proposition 2, when $\phi\left(\frac{1}{n-1}\right)$ lies between $1 / \sqrt{2}-\phi(1)$ and $\phi(1)$, the symmetric star network architecture is Nash stable. On the other hand, according to Proposition 1 , there always exists a $\phi\left(\frac{1}{n-1}\right)$ which is close enough to $\phi(1)$ such that the symmetric star is inefficient. Combining the two results, it follows that, there exist some strength technologies associated with a $\phi\left(\frac{1}{n-1}\right)$ which is large enough so that the Nash (star) network architecture is dominated by the the symmetric complete network. Because the symmetric star dominates every other stars, when the symmetric star architecture is not efficient, no other stars can be. Hence, the efficient network cannot be a star and the tension between efficiency and stability is beyond the class of star architectures. 
Third, it can be shown that if $\phi\left(\frac{1}{n-1}\right)$ approaches $\phi(1)$ and $\phi(1)$ is sufficiently low, then the (non-minimal) complete network will be the unique stable and efficient network. Let $\bar{U} \equiv n(n-1)[2 \phi(1)]$ denote an upper bound on the maximum total utility any feasible network can generate in our setting. Equation (4) suggests that when $\phi\left(\frac{1}{n-1}\right)$ approaches $\phi(1), U\left(g^{s c}\right)$ approaches $\bar{U}$. For any other network which contains indirect connections, the upper-bound of its total utility cannot be higher than $\bar{U}$ because of the presence of decay. Therefore, the symmetric complete network will be the unique efficient architecture if $\phi\left(\frac{1}{n-1}\right)$ reaches its limit of $\phi(1)$. Now note that when $\phi\left(\frac{1}{n-1}\right)$ approaches $\phi(1)$, the utility a single agent can attain approaches $(n-1)[2 \phi(1)]$. A direct link in $g^{s \mathcal{C}}$ will definitely dominate an indirect link in any network if $\phi\left(\frac{1}{n-1}\right)>[2 \phi(1)]^{2}$, which is possible only if $\phi(1)<1 / 4$. In words, the symmetric complete network will be the unique efficient and unique Nash stable architecture when $\phi\left(\frac{1}{n-1}\right) \rightarrow \phi(1)<1 / 4$.

\section{Discussion}

Individuals choose the time and level of effort they wish to expend in different relationships. The more the resources that an individual invests, the more benefits that the individual can derive from it. However, the marginal returns of a unit of investment in a single link can be increasing or decreasing. If investment in a single link is subject to increasing returns, then individuals with limited resources may only invest in a few or even just one link [12,13]. Oppositely, if marginal returns from investing in a single link is decreasing, then diversification becomes a reasonable strategy. Our results confirm the above observation by showing that the assumption of diminishing marginal returns opens up the possibility of the formation of both clustered (such as the symmetric complete network) and non-clustered networks (such as the symmetric star). Moreover, the tension between efficiency and stability may arise across different architectures. We end this paper by discussing how a small-world network does perform better than stars and the complete network in terms of total utility.

Social networks often exhibit the 'small-world' property: short average path length and high level of clustering $[19,20] .^{3}$ Due to the assumption of a convex link strength technology, the efficient and stable communication networks in Bloch and Dutta [12] are minimal and do not lead to a small-world. Similarly, the wheel architecture derived by Deroïan [13] under convex strength technology does not constitute a small-world when $n$ is large. ${ }^{4}$ We have argued that efficient and stable communication networks need not be minimal in the presence of a concave link strength technology and may thus exhibit the small-world property.

Consider the network in Figure 2, the eight agents are divided into two sub-complete networks which are connected by a single link. ${ }^{5}$ We say it is a small-world network because agents belong to the sub-complete network is highly clustered and the distance between any two agents is not longer than three. Fix the structure as appears in the figure, suppose all agents equally distribute their endowments toward their direct links, then the total value of this small-world network will be given by

$$
36 \phi\left(\frac{1}{3}\right)+16 \phi\left(\frac{1}{4}\right)+24 \phi\left(\frac{1}{4}\right)\left[\phi\left(\frac{1}{3}\right)+\phi\left(\frac{1}{4}\right)\right]+36 \phi\left(\frac{1}{4}\right)\left[\phi\left(\frac{1}{3}\right)+\phi\left(\frac{1}{4}\right)\right]^{2}
$$

Assume $\phi(1)=0.45, \phi\left(\frac{1}{3}\right)=0.42, \phi\left(\frac{1}{4}\right)=0.38$ and $\phi\left(\frac{1}{7}\right)=0.32$, then the network in Figure 2 can generate a value of 37.25 while the total value of a symmetric complete network and a symmetric star network with the same size are only 35.84 and 35.68 respectively. Therefore, concavity opens up the

3 Path length is the number of intermediate agents needed in order to reach from one agent to another agents. Clustering roughly measures the likelihood of the presence of a link between a pair of agents who already have a common direct connection.

4 Given a wheel network, if a new direct link is being formed between a pair of agents who are far away from each other, then the average path length of the resulting network will reduce considerably [20]. A sufficiently concave strength technology is likely to lead to such outcome due to the underlying gains from diversification.

5 Similar structures are also studied by [16] based on a network formation model with exogenous link strength. 
possibility of the emergence of small-world network which is not possible when the strength of link is a convex function of the agents' investment. Note that the link connecting the two sub-complete networks is the weakest link among all - $2 \phi\left(\frac{1}{4}\right)$ - but generates half of the total value of the small-world network (removing the link will reduce the total value from 37.25 to $48 \cdot \phi\left(\frac{1}{3}\right)=18.24$ ) which conjecture [1]'s strength of weak tie observation.

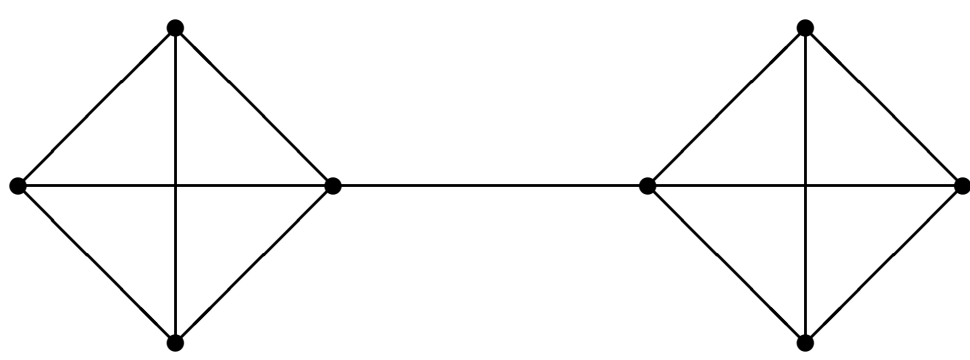

Figure 2. A small-world network.

Acknowledgments: We thank Bhaskar Dutta for his helpful comments on earlier version of this paper. We also thank three anonymous referees for their valuable suggestions and helpful comments. The author especially thank Birendra Rai for his guidance, since the paper was written while the author was a fresh PhD student at Monash University.

Conflicts of Interest: The authors declare no conflict of interest.

\section{Appendix A}

Proof of Lemma A1. The result of budget-exhausting is obvious and hence is omitted. It is the consequence of assumption [Assumption 1] and [Assumption 3] which imply a link strength is non-negative, and no out-side option is available. We now prove the essentiality of the set of efficient and Nash stable networks. Consider a non-essential network $g$. Then there exists a link $i j$ that does not lie along the most reliable path connecting any pair of agents. Therefore, $i$ and $j$ do not communicate through their direct link $i j$ but they do communicate through a sequence of links. Let $i k$ be one of the links in that sequence. Assume without loss of generality $i$ has invested in the link $i j$. Then if $i$ transfers investment from $i j$ to $i k$ and all other investments remain as in $g$ his utility increases and the total utility in $g$ also increases as the withdrawal of investment from a redundant link cannot hurt the utility of other agents.

Proof of Proposition A1. Consider an essential and budget exhausting complete network $g$. Suppose there exist nodes $i, j, k$ such that $\sigma_{i j} \sigma_{j k}>\sigma_{i k}$. Then, $i$ and $k$ will not communicate through their direct link $i k$. Essentiality requires that $i k$ must lie on the most reliable path connecting some pair of agents, say $l$ and $m$. Assume without loss of generality that this path is $(l, \ldots, i, k, \ldots, m)$. Then one can always construct a more reliable path by replacing $(i, k)$ with $(i, j, k)$ and hence contradicts essentiality. Since all the connections are direct links, the total utility in $g$ can be written as $\sum_{i} \sum_{j \neq i}\left[\phi\left(x_{i}^{j}\right)+\phi\left(x_{j}^{i}\right)\right]=$ $2 \sum_{i} \sum_{j \neq i} \phi\left(x_{i}^{j}\right)$. Strict concavity implies that $\sum_{j \neq i} \phi\left(x_{i}^{j}\right)$ is maximized if and only if $x_{i}^{j}=\frac{1}{n-1}$ for all $j \neq i$.

Consider an essential and budget exhausting star network $g$ which is also non-symmetric. Let agent- $i$ be the hub of $g$. Since $g$ is non-symmetric, there exist nodes $j, k$ such that $x_{i}^{j} \neq x_{i}^{k}$. Without loss of generality assume $x_{i}^{j}>x_{i}^{k}$. Let $g^{\prime}$ be the network such that $x_{i}^{\prime j}=x_{i}^{j}-a \geq$ $x_{i}^{\prime k}=x_{i}^{k}+a$ and all other investments remain as in $g$. The increase in total utility in going from $g$ to $g^{\prime}$ is $2\left(\sigma_{i j}^{\prime}-\sigma_{i j}+\sigma_{i k}^{\prime}-\sigma_{i k}\right)\left(1+\sum_{l \neq i, j, k} \sigma_{i l}\right)+2\left(\sigma_{i j}^{\prime} \sigma_{i k}^{\prime}-\sigma_{i j} \sigma_{i k}\right)$, which is strictly positive due to concavity of the strength technology. This implies that $g^{\prime}$ dominates $g$. Fix the strategy 
profile of all the peripherals in $g$ (let the profile be $\overline{\mathbf{x}}_{-i}$ with $x_{j}^{i}=1$ for all $j \in \mathbf{N} \backslash\{i\}$ ), then the set of budget-exhausting stars with $i$ as the hub is formally equivalent to the compact set $S=\left\{\mathbf{x}_{i}: x_{i}^{j} \in[0,1] \forall j \in N \backslash\{i\}\right.$ and $\left.\sum_{j \in \mathbf{N}} x_{i}^{j}=1\right\}$. The total utility for any star $g\left(\mathbf{x}_{i} ; \overline{\mathbf{x}}_{-i}\right)$ is given by $U\left(g\left(\mathbf{x}_{i} ; \overline{\mathbf{x}}_{-i}\right)\right)=2 \sum_{l \neq i} \sigma_{i l}\left(\mathbf{x}_{i} ; \overline{\mathbf{x}}_{-i}\right)+2 \sum_{l, m \neq i ; l \neq m} \sigma_{i l}\left(\mathbf{x}_{i} ; \overline{\mathbf{x}}_{-i}\right) \sigma_{i m}\left(\mathbf{x}_{i} ; \overline{\mathbf{x}}_{-i}\right)$, which is a continuous function of $\mathbf{x}_{i}$ (strict concavity of $\phi$ implies the continuity of $\sigma$ ). Extreme value theorem implies that $U\left(g\left(\mathbf{x}_{i} ; \overline{\mathbf{x}}_{-i}\right)\right)$ reaches a maximum in $S$. As non-symmetric stars have been proven to be dominated, the maximum must be reached for the symmetric star.

Let $\phi(1)$ be $\alpha$ and $\phi\left(\frac{1}{n-1}\right)$ be $\beta$. Define $f(\alpha, \beta, n) \equiv U\left(g^{s S}\right)-U\left(g^{s c}\right)=-(n-2) \beta^{2}+2[(n-1)-$ $(n-2) \alpha] \beta-\left[2 \alpha+(n-2) \alpha^{2}\right]$. For any given $\alpha$ and $n, f$ is a quadratic function of $\beta$. The coefficients and the constant term reveals the fact that $f(\beta ; \alpha, n)$ has a global maximum and cuts the y-axis below zero. Moreover, both roots are positive. We need to show that there exists a root $\beta^{*}<\alpha$ such that $U\left(g^{s c}\right)>U\left(g^{s S}\right)$ for all $\beta \in\left(\beta^{*}, \alpha\right)$. Proving $f(\alpha ; \alpha, n)>0$ is equivalent to proving that the smaller positive root is strictly less than $\alpha$. Solving $f(\alpha ; \alpha, n)$ will give us $2(n-2) \alpha-4(n-2) \alpha^{2}$ which is positive for all $\alpha$ strictly less than half.

Proof of Proposition A2. Pick any agent $i$ from $g^{s c}$. Let $g^{\prime}=g\left(\mathbf{x}_{i}^{\prime}, \mathbf{x}_{-i}\right)$ be a network obtained from $g^{s c}$ in which only $i$ has changed his strategy from $\mathbf{x}_{i}$ to $\mathbf{x}_{i}^{\prime}$. If every connection of $i$ in $g^{\prime}$ is still a direct link, then $i^{\prime}$ s payoff must have decreased because of concavity $\left(u_{i}\left(g^{\prime}\right)=\sum_{j \neq i} \phi\left(x_{i}^{\prime j}\right)+(n-1) \phi\left(\frac{1}{n-1}\right)<\right.$ $2(n-1) \phi\left(\frac{1}{n-1}\right)=u_{i}(g)$ unless $\left.\mathbf{x}_{i}=\mathbf{x}_{i}^{\prime}\right)$. Given $\mathbf{x}_{-i}$, the most reliable indirect connection $i$ can construct in $g^{\prime}$ will have a reliability of $2 \phi\left(\frac{1}{n-1}\right)\left[\phi(1)+\phi\left(\frac{1}{n-1}\right)\right]$. Similarly, the least reliable direct connection between $i$ and some other agent will have a reliability of $\phi\left(\frac{1}{n-1}\right)$. No indirect connection will be possible in $g^{\prime}$ if the weakest possible direct connection is more reliable than the strongest possible indirect connection. Simplifying $2 \phi\left(\frac{1}{n-1}\right)\left[\phi(1)+\phi\left(\frac{1}{n-1}\right)\right]<\phi\left(\frac{1}{n-1}\right)$ will give us the result.

Now we turn to the proof of part (b). By concavity, the hub's best response is to equally split his investment toward all the peripherals and thus has no incentive to deviate from a symmetric star. Let agent 1 be the hub and agent $i$ be any of the peripherals. We consider two cases.

If $i$ chooses not to link to everyone, then the most reliable path connecting $i$ with the hub will serve as the bridge linking $i$ with agents who are not directly connected with $i$. Let $k$ be one such agent and such a path will be either of the form $(i, 1, k)$ or $(i, j, 1, k)$ where $j$ is a peripheral whom $i$ chooses to invest the most. Suppose $(i, j, 1, k)$ is a more reliable path than $(i, 1, k)$, then $x_{i}^{1}=0$ otherwise the resulting network will not be essential. The benefit that $i$ can derive from her connections with $j, 1$ and $k$ is $\phi\left(x_{i}^{j}\right)+\phi\left(x_{i}^{j}\right)\left[\phi(1)+\phi\left(\frac{1}{n-1}\right)\right]+\phi\left(x_{i}^{j}\right)\left[\phi(1)+\phi\left(\frac{1}{n-1}\right)\right]^{2}$. Now consider an alternative deviation such that $i$ invests nothing in $j$ but invests an equal amount of $x_{i}^{j}$ in the hub. Then the benefit that $i$ can derive from her connections with $j, 1$ and $k$ will become $\left[\phi\left(x_{i}^{j}\right)+\phi\left(\frac{1}{n-1}\right)\right]+2\left[\phi\left(x_{i}^{j}\right)+\phi\left(\frac{1}{n-1}\right)\right]\left[\phi(1)+\phi\left(\frac{1}{n-1}\right)\right]$. Therefore, $(i, j, 1, k)$ cannot be a more reliable path than $(i, 1, k)$. Therefore, when $i$ deviates in such a way, the benefits he can derive from the hub and the other peripherals whom he chooses not to directly link with will be lowered when $i 1$ is weakened. Thus if the strength of an indirect connection in a symmetric star is stronger than direct connection $i$ has newly established, such a deviation cannot be profitable. Note that a direct connection from a peripheral to another peripheral cannot be stronger than $\phi(1)<\frac{1}{2}$ and the strength of an indirect connection in a symmetric star is $\left[\phi(1)+\phi\left(\frac{1}{n-1}\right)\right]^{2}$, the result follows.

Now suppose $i$ chooses to directly link to all the other agents, then, by concavity, his best response will be $\left(\frac{1}{n-1}, \ldots, 0, \ldots, \frac{1}{n-1}\right)$. As a result, $i$ 's connection with the hub would be weakened from $\phi(1)+\phi\left(\frac{1}{n-1}\right)$ to $2 \phi\left(\frac{1}{n-1}\right)$. Similar to the previous case, if the strength of an indirect connection in a symmetric star is stronger than direct connection $i$ has newly established, such a deviation cannot be profitable. The direct connection from a peripheral to another peripheral is $\phi\left(\frac{1}{n-1}\right) \leq \phi(1)<\frac{1}{2}$ and the strength of an indirect connection in a symmetric star is $\left[\phi(1)+\phi\left(\frac{1}{n-1}\right)\right]^{2}$, the result follows. 


\section{References}

1. Granovetter, M. The strength of weak ties. Am. J. Sociol. 1973, 78, 1360-1380.

2. Coleman, J.; Katz, E.; Menzel, H. Medical Innovation: A Diffusion Study; Bobbs-Merrill Company: New York, NY, USA, 1966.

3. Bailey, N. The Mathematical Theory of Infectious Diseases and Its Applications; Charles Griffin: London, UK, 1975.

4. Enemark, D.; McCubbins, M.; Weller, N. Knowledge and networks: An experimental test of how network knowledge affects coordination. Soc. Netw. 2012, 36, 122-133.

5. Goyal, S. Connections: An Introduction of the Economics of Networks; Princeton University Press: Princeton, NJ, USA, 2007.

6. Jackson, M. Social and Economic Networks; Princeton University Press: Princeton, NJ, USA, 2008.

7. Jackson, M.; Wolinsky, A. A strategic model of social and economic networks. J. Econ. Theory 1996, 71, 44-74.

8. Bala, V.; Goyal, S. A noncooperative model of network formation. Econometrica 2000, 68, 1181-1229.

9. Dutta, B.; Mutuswami, S. Stable networks. J. Econ. Theory 1997, 76, 322-344.

10. Dutta, B.; Jackson, M. The stability and efficiency of directed communication networks. Rev. Econ. Des. 2000, 5, 251-272.

11. Jackson, M.; van den Nouweland, A. Strongly stable networks. Games Econ. Behav. 2005, 51, 420-444.

12. Bloch, F.; Dutta, B. Communication networks with endogenous link strength. Games Econ. Behav. 2009, 66, 39-56.

13. Deroïan, F. Endogenous link strength in directed communication networks. Math. Soc. Sci. 2009, 57, 110-116.

14. Feri, F.; Meléndez-Jiménez, M. Coordination in evolving networks with endogenous decay. J. Evol. Econ. 2013, 23, 955-1000.

15. Harmsen-an Hout, M.; Herings, P.; Dellaert, B. Communication network formation with link specificity and value transferability. Eur. J. Oper. Res. 2013, 229, 199-211.

16. Jackson, M.; Rogers, B. The economics of small worlds. J. Eur. Econ. Assoc. 2005, 3, 617-627.

17. Baumann, L. Time allocation in friendship networks. Available online: https://papers.ssrn.com/sol3/ papers.cfm?abstract_id=2533533 (accessed on 8 December 2016).

18. Salonen, H. Reciprocal equilibria in link formation games. Czech Econ. Rev. 2014, 9, 169-183.

19. Wasserman, S.; Faust, K. Social Network Analysis: Methods and Applications; Cambridge University Press: Cambridge, UK, 1994.

20. Watts, D.; Strogatz, S. Collective dynamics of 'small-world' networks. Nature 1998, 393, 440-442.

(C) 2016 by the author; licensee MDPI, Basel, Switzerland. This article is an open access article distributed under the terms and conditions of the Creative Commons Attribution (CC-BY) license (http://creativecommons.org/licenses/by/4.0/). 